\title{
Dans le vent du vocabulaire
}

Mme Denise Pumain

\section{Citer ce document / Cite this document :}

Pumain Denise. Dans le vent du vocabulaire. In: Espace géographique, tome 16, n³, 1987. pp. 214-215;

doi : https://doi.org/10.3406/spgeo.1987.4249

https://www.persee.fr/doc/spgeo_0046-2497_1987_num_16_3_4249

Fichier pdf généré le 02/04/2018 


\section{Les mots de la géographie}

\section{DANS LE VENT DU VOCABULAIRE...}

La littérature géographique emploie parfois des mots du langage courant pour désigner des objets qui, par suite du développement des connaissances ou des outils, se chargent d'une signification plus précise ou plus particulière. Il devient à un certain moment nécessaire de faire le point sur ces glissements sémantiques, de façon à éviter la prolongation d'ambiguïtés pouvant conduire à des malentendus.

Ainsi, l'adjectif "dynamique " est-il associé dans le vocabulaire courant à l'idée d'un développement rapide et d'une attitude active: une région dynamique, le dynamisme d'un maire... Son emploi de plus en plus fréquent dans l'expression "modèle dynamique " nous incite à préciser le sens de ce second usage. Nous le faisons ici seulement pour des modèles qui ont des transcriptions mathématiques, et qui sont des modèles de systèmes spatiaux.

Un système spatial est défini comme un ensemble d'unités géographiques en interaction, dont chacune est décrite par un petit nombre de variables d'état. Les équations du modèle mettent en relation ces variables et des paramètres. Dans la pratique, et par approximation, les variables sont des descripteurs du système susceptibles de changer de valeur (dynamique rapide), tandis que les paramètres correspondent à des descripteurs qui peuvent être supposés permanents (constantes) ou variant très lentement (dynamique lente).

On distingue trois types de modèles, selon la nature des relations qu'ils décrivent.

- Les modèles statiques décrivent des configurations spatiales (de phénomènes implantés localement ou de déplacements). Comme les configurations repérables ont une forte probabilité de correspondre à des états stables, on dit parfois qu'il s'agit de " modèles d'équilibre ". Par exemple, les modèles de Weber, de Christaller, d'Alonso, le modèle de gravité sont des modèles statiques. Leur forme générale est : variable d'état $=(\mathrm{f})$ variables d'états et paramètres.

- Les modèles cinétiques dits encore modèles de processus décrivent la variation temporelle d'une configuration. Ces modèles d'évolution permettent de calculer le changement sans l'expliquer. Les modèles qui dessinent des projections, les modèles utilisant les chaînes de Markov sont des exemples de modèles cinétiques. Leur forme générale est :

variable d'état au temps $t+1=(f)$ variables d'état $a u$ temps $\mathrm{t}, \mathrm{t}-1$... et paramètres.

- Les modèles dynamiques décrivent aussi le changement dans une configuration spatiale, mais en introdui- sant dans l'équation différentielle des modèles continus, ou dans l'équation aux différences des modèles de type forrestérien par exemple, les causes de la variation temporelle. Les modèles dits non-linéaires incluent toujours au moins une boucle de rétroaction : le changement temporel dépend du niveau atteint par les variables d'état. Leur forme générale est: taux d'évolution de la variable d'état $=(\mathrm{f})$ variables d'état et paramètres.

Ces trois types de modèles sont hiérarchisés par emboîtement: le modèle dynamique peut simuler plusieurs types de trajectoires et le passage de l'une à l'autre (phénomène de bifurcation dans les zones d'instabilité), le modèle cinétique simule une trajectoire, c'est-à-dire une variation d'une configuration au cours du temps, le modèle statique simule une structure qui est un moment de cette évolution et qui correspond le plus souvent à un état stable d'un modèle dynamique.

Les connotations associées à chaque type de modèle peuvent être résumées dans le tableau suivant :

\begin{tabular}{|c|c|c|c|}
\hline $\begin{array}{c}\text { Type } \\
\text { de modèle }\end{array}$ & $\begin{array}{l}\text { Relations } \\
\text { décrites }\end{array}$ & Référence & Objectif \\
\hline $\begin{array}{l}\text { Statique } \\
\text { Cinétique } \\
\text { Dynamique } \\
\text { (forces } \\
\text { exogènes) } \\
\text { Auto-dynamique } \\
\text { (forces } \\
\text { endogènes) }\end{array}$ & $\begin{array}{l}\text { Structure } \\
\text { Processus } \\
\text { Régulation } \\
\text { (changement } \\
\text { de processus) } \\
\text { Auto-organisation } \\
\text { (changement } \\
\text { des régulations) }\end{array}$ & $\begin{array}{l}\text { Equilibre } \\
\text { Trajectoire } \\
\text { Génétique } \\
\text { Création }\end{array}$ & $\begin{array}{l}\text { Descriptif } \\
\text { ou explicatif } \\
\text { Descriptif } \\
\text { Explicatif } \\
\text { Explicatif }\end{array}$ \\
\hline
\end{tabular}

Si cette terminologie emporte un large consensus, le vocabulaire qui différencie les modèles à l'intérieur de la catégorie dynamique est plus hésitant. On distingue les modèles "agrégés" ou d'échelle "macro", formulés directement pour des variables d'état définies à l'échelon de chaque unité géographique, et les modèles " désagrégés " ou d'échelle "micro ", qui déduisent les comportements de ces agrégats d'une modélisation des comportements individuels des éléments du système. Certains modèles correspondent à un seul niveau d'organisation, tandis que les modèles dits hiérarchiques en simulent deux ou plusieurs. Les modèles dynamiques d'auto-organisation seraient les plus performants car ils sont supposés contenir toutes les forces qui causent l'évolution du système et les changements de ces forces. Fxistent.ils en pratique? D'aucuns nomment cela le 
" modèle de la grande sœur " (celle qui change les règles du jeu en fonction de l'évolution du jeu), ou bien faut-il préférer dire: "modèle de Big Brother"?

Cette proposition de stabilisation du vocabulaire, qui appelle des rétroactions, est un état provisoire dans l'évolution de la pensée collective, sous l'effet d'une forte dynamique interne, à l'équipe P.A.R.I.S... Y ont contribué : François Durand-Dastès. France Guérin. Denise Pumain, Bertrand Roehner. Léna Sanders. Thérèse Saint-Julien, Peter Winiwarter.- P.c.c. Denise PUMAIN, Université de Paris-XIII.

\section{LE PLEXAGE DE L'ESPACE}

En découvrant, sous la diversité des distributions et des configurations spatiales, des formes élémentaires qu'il a proposé d'appeler chorèmes, et en montrant que leur agencement était régi par un petit nombre de règles topologiques, R. Brunet a doté la géographie d'un outil conceptuel remarquable.

C'est ainsi qu'il a été amené à distinguer deux modalités fondamentales d'organisation de l'espace : le maillage et le quadrillage. La pertinence du contenu de ces deux notions est indiscutable, tout de même que l'appellation de la première. Mais le terme de quadrillage prête, à mon sens, à discussion, ce d'autant plus que certains esprits, pourtant au fait des définitions qui en ont été données dans "L'Espace Géographique ", font parfois la confusion et intervertissent les deux termes. Je suggère que le terme de quadrillage soit remplacé par celui de plexage. Je m'en explique brièvement.

Le maillage est un terme très bien choisi pour qualifier la logique première de contrôle du terrain : sa "re-connaissance ", sa "dé-limitation", sa " dé-nomination ", accompagnent toute entreprise d'action sur l'espace. Il faut jeter sur lui un filet pour en saisir le contenu. Plus celui-ci est lourd, c'est-à-dire de haute densité, plus le maillage est fin : la maille administrative de la vieille Europe est plus serrée que celle du sertão ou de la Sibérie.

La seconde logique d'action sur l'espace est plus dynamique. Elle est celle des relations entre un centre et sa périphérie. Les voies de communication matérialisent au sol (sans oublier les airs et les mers) ce qui est encore, comme $R$. Brunet l'a montré, une relation de pouvoir et de domination. Peut-être cet aspect des choses a-t-il inspiré ce terme de quadrillage, auquel je trouve pour ma part une connotation un peu trop militaire, une idée de contrôle itératif, qui favorise la confusion avec le maillage.

Par ailleurs le terme de quadrillage ne pourrait s'appliquer en toute rigueur, je le dis... carrément. qu'au réseau régulier des voies disposées en grille ou en damier. Il est certes effectivement assez fréquent dans les villes. Du moins dans celles qui ont été construites à la hâte ou diune seule volée. soit par nécessité militaire et sur dessin préconçu, soit par ćconomic d'arpentage et de voierie. On peut le trouver aussi en dehors des villes, soit dans les régions d'aménagement hyddraulique, rizières et polders, soit dans le réseau des chemins ruraux calés sur le parcellaire, sur la cadastration romaine en particulier, soit encore, mais à une autre échelle, et avec plus de concessions à la topographie, dans les réseaux d'autoroutes (Interstate aux Etats-Unis). Le réseau quadrillé imprime à la desserte de l'espace une organisation égalitaire, exhaustive et monotone, qui peut convenir à l'exploitation des ressources agricoles d'un espace isotrope.

Mais il convient mal aux relations tous azimuths d'un centre avec sa périphérie ou avec d'autres centres. Dans une ville quadrillée les relations obliques comportent soit un itinéraire simplifié, ne comportant qu'un seul coude, mais au prix d'un énorme détour, un allongement de $41 \%$ de la distance parcourue, soit les louvoiements d'une loxodromie dispendieuse en ralentissements, ceux qu'im. posent les courbes des trèfles routiers ou que provoquent les correspondances ferroviaires.

N'est pas fou qui cherche la diagonale, ou la tangente... Voyez Broadway. Les branchés suivent le Trunkway. Ailleurs les maîtres du régime foncent sur la " magistrale ", tandis que les canards boîteux hésitent à la patte d'oie. Si la ville vaut le voyage, l'étoile de la place, encombrée, mérite un détour : en lieu et place du Trocadéro. vous prendrez la rocade. Humaine comédie des boulevards, des avenues, des venelles et des déviations. L'espace donne de la voie dans toutes les directions.

Symbole de notre destinée, la croisée des chemins a son implacable dessin. Trop simple. Multiples destinations ont bien souvent embranchements tortueux au long des voies de pénétration, entreprises de diramations, nœuds gordiens des échangeurs tranchant sur l'ordre des champs, aiguillages et rebroussements, entrelacs et anastomoses.

Comme associées en direct par multiplex. Ariane et Pénélope me suggèrent donc d'en découdre avec le quadrillage et. sans perdre le fil et sans complexe. de parler du plexage de l'espace.- Pierre RIQUET, Lniversité de Paris-I, novembre 1987. 